\title{
Efecto del Recubrimiento Comestible en las Propiedades de Trozos de Batata (Ipomoea Batatas Lam) Fritos por Inmersión. Parte 1: Textura
}

\author{
Adriana González, Armando Alvis* y Guillermo Arrázola \\ Universidad de Córdoba. Programa de Ingeniería de Alimentos. Facultad de Ingenierías. Grupo de \\ Investigación Procesos y Agroindustria de vegetales. Carrera 6 № 76-103, Córdoba, Colombia. \\ (e-mail: aalvis2@hotmail.com) \\ * autor a quien debe ser dirigida la correspondencia
}

Recibido Jun. 9, 2014; Aceptado Ago. 20, 2014; Versión final recibida Sep. 1, 2014

\begin{abstract}
Resumen
En este estudio se caracterizaron las variedades de batata roja y amarilla cultivadas en el departamento de Córdoba. La caracterización bromatológica consistió en la medida del contenido de humedad, grasa, proteína, ceniza, fibra, azúcares reductores y carbohidratos totales. La batata roja, por su menor contenido de azúcares reductores, fue seleccionada para un estudio sobre freído por inmersión. Para evaluar la textura de los trozos de batata fritos se midió la fuerza de corte y se realizó un análisis de perfil de textura utilizando un texturómetro TA.XTPlus. El recubrimiento utilizado fue carboximetilcelulosa y las temperaturas de fritura fueron de 150,170 y $190 \stackrel{\circ}{-}$. Estas no afectaron significativamente a las variables de textura como dureza, adhesividad, elasticidad, gomosidad y masticabilidad, pero si afectaron la cohesividad del producto. La fuerza de corte del producto sólo se vio afectada por el uso del recubrimiento.
\end{abstract}

Palabras clave: batata, fritura, caracterización bromatológica, textura, carboximetilcelulosa

\section{Effect of Edible Coating on the Properties of Sweet Potato Slices (Ipomoea Batatas Lam) Cooked by Deep-Fat Frying. Part 1: Texture}

\begin{abstract}
In this study varieties of red and yellow sweet potatoes grown in the department of Córdoba were characterized. Bromatological characterization consists in measuring the content of moisture, fat, protein, ash, fiber, reducing sugars and total carbohydrates. Red sweet potatoes, for their lower content of reducing sugars, were selected for a study of deep fat frying. To evaluate the texture of fried potato chips the cutting force was measured and texture profile analysis was performed using a TA.XTplus texturometer. The edible coating used was carboxymethylcellulose and the frying temperatures were 150,170 and $190^{\circ} \mathrm{C}$. These temperatures did not have significant effect on the texture variables such as hardness, adhesiveness, springiness, gumminess and chewiness, but they affected cohesiveness of the product. The cutting force of the product was affected only by the use of the coating.
\end{abstract}

Keywords: sweet potato, deep fat frying, bromatological characterization, texture, carboxymethylcellulose 


\section{INTRODUCCIÓN}

La batata (Ipomoea Batatas Lam) es un tubérculo que se siembra en Suramérica y se reconoce como un producto ligado a todas las épocas y a la cultura campesina; sin embargo, en Colombia su cultivo se encuentra aún en estado primitivo (FAO, 2010). En el año 2012 se produjeron más de 103 millones de toneladas en el mundo, de los cuales el $70,91 \%$ es producido por China (FAO 2014). En América Latina para este mismo año se destaca la producción anual en Brasil con $479.425 \mathrm{t}$. Argentina con $400.000 \mathrm{t}$ y Perú con 304.006 t (FAO 2014). Colombia en el año 2012 reporta una producción total de 156 t en un área de $13 \mathrm{Ha}$ en el departamento de Sucre con una participación nacional del $100 \%$. Córdoba reporta una producción representativa en el año 2008 con una producción de 25 t en $5 \mathrm{Ha}$ (Agronet 2014). La batata es un cultivo muy importante y valioso en el mundo, su potencial en la seguridad alimentaria y en el bienestar mundial es bien reconocido gracias a estudios que muestran la importancia de su procesamiento, utilización, funcionalidad, valor nutritivo y características saludables (Waramboi et al., 2011).

La textura es un atributo sensorial de importancia suprema para la preferencia de un producto frito y es un parámetro crítico en la calidad final del producto (Kita et al., 2007). Se espera que un producto frito de buena calidad tenga una corteza crujiente entre 1 y $2 \mathrm{~mm}$ como un indicador de frescura y alta calidad, y un centro cocido, húmedo y suave (Sanz et al., 2007). El análisis de perfil de textura, TPA por sus siglas en inglés, es una simulación de la masticación de una muestra por medio de un equipo analizador de textura. Consiste en comprimir un alimento del tamaño de un mordisco para imitar la acción de los dientes e incluye el análisis de diferentes características de textura de una muestra (Taiwo y Baik 2007; Hleap y Velasco, 2010; Tavera-Quiroz et al.,2012) y se representan gráficamente como se muestra en la figura 1.

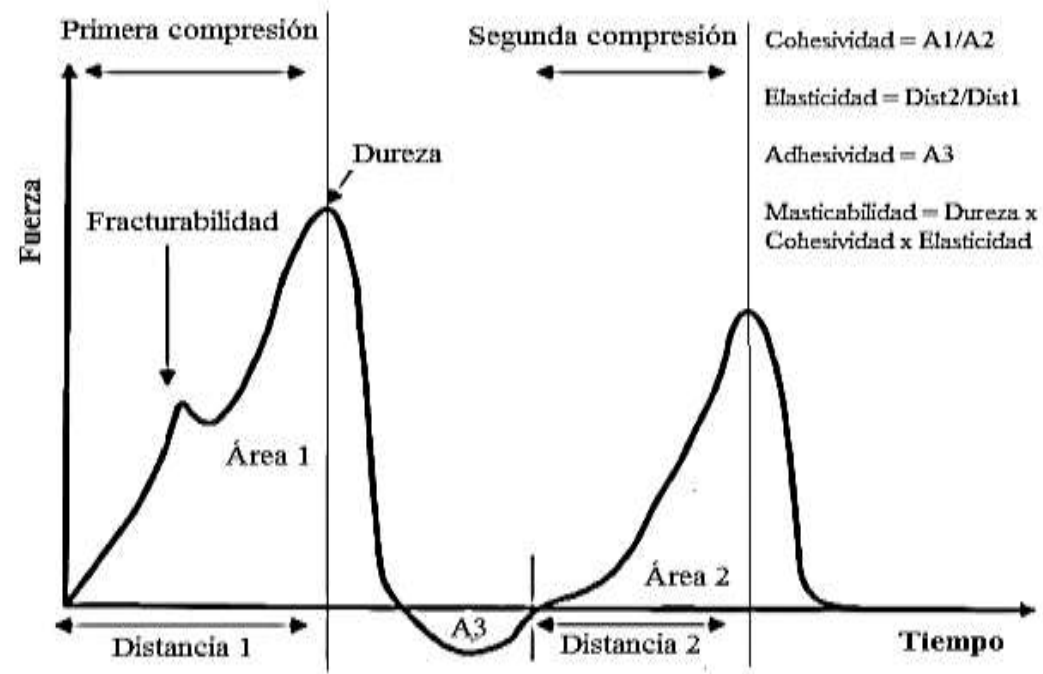

Fig. 1. Gráfica general del análisis de perfil de textura TPA (Hleap y Velasco, 2010)

Fracturabilidad: Es el pico inicial de fuerza durante la primera compresión. Se refiere a la dureza con la cual el alimento se desmorona, cruje o revienta. Se expresa en unidades de fuerza, generalmente Newton.

Dureza: Es la fuerza máxima obtenida durante la primera parte de compresión, imitando el primer mordisco. Se refiere a la fuerza requerida para comprimir un alimento entre los molares o entre la lengua y el paladar. Se expresa en unidades de fuerza, generalmente Newton.

Cohesividad: Es la razón entre el área de fuerza positiva durante la segunda compresión y el área durante la primera compresión. Indica la habilidad de soportar rotura bajo compresión, representa la fuerza con la que están unidas las partículas. Es adimensional y un valor igual a uno indica que la muestra se recupera completamente luego de la primera compresión.

\section{Cohesividad $=$ Área2/Área1}

Adhesividad: Es el área de fuerza negativa en el primer mordisco y representa el trabajo requerido para superar las fuerzas atractivas entre la superficie del alimento y la superficie de otros materiales con los que el alimento entra en contacto. Se mide en unidades de trabajo, generalmente Joule.

Gomosidad: Es el producto de la dureza por la cohesividad. Simula la energía requerida para desintegrar un alimento semisólido para que éste se pueda tragar. Se expresa en unidades de fuerza, Newton. 


\section{Gomosidad $=$ Dureza $\times$ Cohesividad}

Elasticidad: Se relaciona con la altura a la que el alimento se recupera durante el lapso de tiempo entre el final del primer mordisco y el inicio del segundo. Mide cuánta estructura original del alimento se ha roto por la compresión inicial. Es adimensional.

Elasticidad $=$ Distancia 2/ Distancia 1

Masticabilidad: Es la medida de la energía requerida para masticar un sólido y desintegrarlo hasta que se pueda tragar. Es igual al producto de la gomosidad por la elasticidad. Se expresa en unidades de masa, generalmente $\mathrm{kg}$.

\section{Masticabilidad $=$ Dureza $\times$ Cohesividad $\times$ Elasticidad}

\section{MATERIALES Y MÉTODOS}

Se seleccionaron las batatas de las variedades amarilla y roja producidas en el departamento de Córdoba, obtenidas del banco de germoplasma de CORPOICA Turipana. Se recolectaron aproximadamente 20 kilogramos de cada muestra con tamaños y pesos semejantes. Las batatas se conservaron enteras a una temperatura aproximada de $26{ }^{\circ} \mathrm{C}$ y humedad del ambiente durante el desarrollo de la investigación.

La caracterización bromatológica de las dos variedades de batata se realizó por triplicado, mediante los métodos de la AOAC (2003). Se caracterizaron los trozos de batata cruda, peladas y cortadas manualmente. El contenido de humedad: Método 925.09 de la AOAC, contenido de grasa: Método 920.39 de a AOAC., contenido de proteína: Método 920.87 de la AOAC., contenido de ceniza: Método 923.03 de la AOAC., contenido de fibra: Método 962.09 de la AOAC., Azúcares reductores: Manual de prácticas de análisis de alimentos (2010) y el contenido de carbohidratos totales se estimó por diferencia, ecuación 5.

Porcentaje de Carbohidratos Totales = $100-(\%$ humedad + \%grasa + \%proteína + \%ceniza + \%fibra)

Para analizar las diferencias entre la composición bromatológica de cada variedad se definió un intervalo de confianza del $95 \%$. El número de repeticiones por tratamiento fueron tres. Las batatas se conservaron enteras a una temperatura aproximada de $26{ }^{\circ} \mathrm{C}$ y humedad del ambiente durante el desarrollo de la investigación. Las unidades de batata fueron lavadas con abundante agua y peladas y cortadas manualmente con las dimensiones de $1 \mathrm{~cm} \times 1 \mathrm{~cm} \times 5 \mathrm{~cm}$. Todas las muestras fueron sometidas a escaldado en una solución acuosa al $0.5 \%$ de cloruro de calcio en ebullición durante 5 minutos y se secaron con papel absorbente por un minuto. La mitad de las muestras fueron sometidas al pretratamiento de recubrimiento comestible y la otra mitad fue sometida a freído directamente. El pretratamiento de recubrimiento comestible se realizó sumergiendo las muestras en una solución acuosa de carboximetil celulosa al $0.5 \%$ a temperatura ambiente durante un minuto. Se dejaron en escurridor metálico mientras se secaban con un secador manual con velocidad de $28.53 \mathrm{mph}$ ó $12.75 \mathrm{~m} / \mathrm{s}$ y temperatura aproximada de 98 oC durante seis minutos. El proceso de freído se realizó con aceite de palma en una freidora a tres temperaturas: 150,170 y $190{ }^{\circ} \mathrm{C}$, y dos pretratamientos: escaldado y escaldado más recubrimiento comestible.

El análisis de fuerza máxima de corte se realizó mediante un texturómetro con instrumento de aluminio en forma de cuña, con $30^{\circ}$ de ángulo de corte de y $15 \mathrm{~mm}$ de ancho, a una velocidad de $40 \mathrm{~mm} / \mathrm{s}$ sobre una superficie plana. La textura de la batata se midió en todas las muestras fritas a un tiempo definido para cada temperatura suficiente para alcanzar un contenido de humedad promedio de $49.32 \% \pm 1,27 \%$, así: para $150^{\circ} \mathrm{C}$ se definió un tiempo de freído de 300 segundos, para $170{ }^{\circ} \mathrm{C}$ el tiempo fue de 180 segundos y para $190 \stackrel{\circ}{\circ}$ el tiempo fue de 120 segundos.

La prueba de TPA se realizó mediante dos compresiones simulando la mordida humana, utilizando un émbolo de $3.8 \mathrm{~cm}$ de diámetro con base de varilla de $2 \mathrm{~cm}$ de diámetro. En el análisis de perfil de textura TPA se determinaron las características de dureza, cohesividad, adhesividad, gomosidad, elasticidad y masticabilidad. Para el análisis de la influencia de la temperatura y el recubrimiento sobre todas las variables medidas se aplicó un diseño de experimentos factorial, donde se tomaron como tratamientos las seis combinaciones de los factores temperatura y recubrimiento, cuyos niveles fueron 150,170 y $190{ }^{\circ} \mathrm{C}$ para la temperatura y ausencia (0) y presencia (0.5) para el recubrimiento. El número de repeticiones por tratamiento dependió de la variable. 


\section{RESULTADOS Y DISCUSIÓN}

Los resultados de la caracterización bromatológica de las dos variedades de batata se muestran en la tabla 1 , donde se puede observar que la variedad de batata roja tiene menor contenido de humedad y de proteínas, mientras que tiene mayor contenido de grasa, ceniza y carbohidratos, comparándola con la variedad amarilla.

Tabla 1. Composición bromatológica de batata roja y amarilla. *Diferencia significativa

\begin{tabular}{|l|c|c|c|c|}
\hline & \multicolumn{2}{|c|}{ Roja } & \multicolumn{2}{c|}{ Amarilla } \\
\hline Composición & Prom (\%) & $\pm S D$ & Prom (\%) & $\pm S D$ \\
\hline \% humedad & $66,17^{*}$ & 0,68 & $73,14^{*}$ & 0,39 \\
\hline \% grasa & $3,28^{*}$ & 0,16 & $0,54^{*}$ & 0,03 \\
\hline \% proteína & $1,95^{*}$ & 0,12 & $2,19^{*}$ & 0,07 \\
\hline \% ceniza & 1,24 & 0,10 & 0,15 & 0,03 \\
\hline \% fibra & 3,39 & 0,08 & 3,28 & 0,07 \\
\hline \% carbohidratos & $23,99^{*}$ & 0,74 & $20,70^{*}$ & 0,31 \\
\hline \% azúcares reductores & $26,45^{*}$ & 0,92 & $45,77^{*}$ & 0,43 \\
\hline
\end{tabular}

Estos resultados son similares a los obtenidos por Pletsch (2006), quien reporta la humedad de la variedad amarilla en $67.7 \%$ y la de la variedad colorada en $77 \%$, la proteína de ambas las reporta en $2.2 \%$ y $2 \%$, la ceniza en $1 \%$ y $0.6 \%$, para amarilla y colorada, respectivamente. Aunque estos valores son similares con los obtenidos, parecen también contradictorios, pero esto se justifica porque existen muchas variedades de batata clasificadas según el color, gran variedad de amarillas y rojas de diferentes formas y composiciones (Arrieta y Gutiérrez, 1995) que dependen de las condiciones bajo las que se cultive (An et al., 2003). Los porcentajes de carbohidratos también se encuentran cerca del rango definido para las batatas reportado por Iwe et al. (1998) entre 80 y $90 \%$. Albán y Cadavid (2006) reportaron los rangos de porcentaje de composición para la batata; en ellos se indica que los valores de almidón se encuentran entre 30 y $80 \%$, las proteínas entre 1.2 y $10.0 \%$ y las cenizas entre 0.6 y $4.5 \%$ y los resultados obtenidos en este estudio se encuentran dentro de los valores esperados. Otros valores similares para la batata son los reportados por Zhang et al. (2011) para carbohidratos en $34.12 \%$, humedad $61.5 \%$, proteína $0.7 \%$, ceniza $1.61 \%$, fibra $2.2 \%$ y grasa $0.2 \%$; y los reportados por Farinu y Baik (2007) para carbohidratos en $26.3 \%$, humedad 69.9 $\%$, proteína $1.7 \%$, ceniza $1.0 \%$, fibra $0.7 \%$ y grasa $0.4 \%$.

La selección de la variedad de batata que se utilizó en el proceso de freído se realizó teniendo en cuenta el menor contenido de azúcares reductores. Los resultados del porcentaje de azúcares reductores obtenidos se muestran en la tabla 1 y para las dos variedades estudiadas son $26.45 \pm 0.92 \%$ y $45.77 \pm 0.43 \%$, para batata roja y amarilla, respectivamente. Además, para un intervalo de confianza del $95 \%$, el porcentaje medio de azúcares reductores se encuentra entre $25.75 \%$ y $27.16 \%$ para la variedad roja y entre $45.44 \%$ y $46.10 \%$ para la variedad amarilla. Estos datos indican que el menor contenido lo tiene la batata roja, por esta razón fue seleccionada como la variedad a utilizar en el estudio del freído por inmersión, recordando que estos azúcares no son deseables en gran cantidad porque participan en la reacción de Maillard y en la formación de acrilamida (Pedreschi et al., 2007; Durán et al., 2007; Mestdagh et al., 2008). Estos resultados también son mayores a los reportados por Pletsch (2006) de $4.8 \%$ para la variedad amarilla y $6.3 \%$ para la colorada; sin embargo, se encuentran cerca del rango reportado por Albán y Cadavid (2006) entre 5 y $38 \%$. Sin embargo, se utilizan los procesos de escaldado para lixiviar azúcares solubles y evitar el pardeamiento (Mai Tran et al., 2007). Los valores promedios para todas las variables de textura se muestran en la tabla 2.

\section{Fuerza de Corte}

El análisis de la fuerza de corte obtenida experimentalmente para los diferentes tratamientos indicó que hubo un efecto altamente significativo del recubrimiento pero no hubo efecto significativo de la temperatura de freído ni de la interacción de los dos factores analizados. Las muestras con recubrimiento tuvieron una fuerza de corte mayor que las muestras sin recubrimiento; esto se debe a que el recubrimiento pudo disminuir la porosidad en las muestras y proporcionar una superficie más homogénea gracias a la fina capa del recubrimiento (Varela y Fiszman, 2011), mientras que la superficie de la muestra sin recubrimiento se caracteriza por ser más porosa y con más grietas que la hacen más susceptibles a la fractura. 
Tabla 2. Valores promedios para las variables de textura de los trozos de batata fritos.

\begin{tabular}{|c|c|c|c|c|c|c|c|}
\hline \multirow{2}{*}{ Tratamiento } & \multicolumn{7}{|c|}{ Características de textura } \\
\cline { 2 - 8 } & FC & Dur. & Adh. & Coh. & Elas. & Gom.90 & Mast. \\
\cline { 2 - 8 } & $\mathrm{N}$ & $\mathrm{g}$ & $\mathrm{g.s}$ & $\%$ & $\%$ & & $\mathrm{G}$ \\
\hline $150 \mathrm{SR}$ & 29,9 & $1641,3^{*}$ & $-0,7^{*}$ & 0,37 & 64,0 & 464,2 & 266,5 \\
\hline $170 \mathrm{SR}$ & 30,1 & $1892,5^{*}$ & $-3,3$ & 0,52 & 61,7 & 1001,7 & 660,9 \\
\hline $190 \mathrm{SR}$ & 30,2 & $1000,3^{*}$ & $-3,4$ & 0,51 & 62,8 & 458,8 & 266,3 \\
\hline $150 \mathrm{CR}$ & 38,0 & $2160,3^{*}$ & $-1,7^{*}$ & 0,44 & 62,5 & 836,7 & 603,4 \\
\hline $170 \mathrm{CR}$ & 38,8 & $1879,5^{*}$ & $-2,9$ & 0,53 & 61,6 & 771,2 & 475,1 \\
\hline $190 \mathrm{CR}$ & 31,1 & $1481,0^{*}$ & $-2,5$ & 0,53 & 62,4 & 791,5 & 506,0 \\
\hline
\end{tabular}

En la Tabla 2, FC significa fuerza de corte, Dur. dureza, Adh. adhesividad, Coh. cohesividad, Elas. elasticidad, Gom. gomosidad y Mast. masticabilidad. a diferentes temperaturas (150, 170 y $190 \stackrel{\circ}{ }$ ) $) \sin$ recubrimiento $(\mathrm{SR})$ y con recubrimiento $(\mathrm{CR}){ }^{*}$ Diferencias significativas

Con respecto a la temperatura, se puede decir que no afecta la fuerza de corte y a la dureza y adhesividad, y no a las otras características de textura que se analizan más adelante como la elasticidad, gomosidad y masticabilidad, debido a que éste factor se relaciona directamente con el tiempo de freído y juntos controlan las transformaciones y las características del producto final (Vitrac et al., 2002). En nuestro caso, los mayores tiempos de fritura se utilizaron para las temperaturas menores del aceite para alcanzar el mismo contenido de humedad y características finales. En estudios similares como los realizados por Gołubowska (2005) y Lisińska y Gołubowska (2005) se obtuvieron valores para la fuerza máxima de corte en trozos de papa fritas de $13 \mathrm{~N}$, pero utilizando velocidades de corte de $250 \mathrm{~mm} / \mathrm{s}$ comparados con los valores promedios entre 29.9 y $38.8 \mathrm{~N}$ a una velocidad de $1 \mathrm{~mm} / \mathrm{s}$ utilizados en este estudio.

\section{Análisis de perfil de textura TPA}

El análisis de perfil de textura se enfoca en seis variables que se analizan a continuación: dureza, adhesividad, cohesividad, elasticidad, gomosidad y masticabilidad.

Los valores de la dureza de todos los tratamientos analizados presentaron diferencia significativa; no se observaron efectos de la temperatura ni del recubrimiento ni de la interacción de estos factores. Esto significa que la fuerza requerida para comprimir los productos de batata fue estadísticamente igual entre todos los tratamientos. Este resultado es alentador porque indica que el uso del recubrimiento no afecta esta variable. Aunque no se analizó la dureza del producto crudo, sí se pudo evidenciar mediante la masticación que el escaldado permitió reducir la dureza del producto crudo debido a la pérdida de la firmeza que se presenta por la sustancial disolución, polimerización y aparente destrucción de las paredes celulares (Gołubowska, 2005; Lisińska y Gołubowska, 2005; Taiwo y Baik, 2007; Alvis et al., 2008) que dependen de la presencia de sustancias pécticas que son parte del material intercelular.

En este punto es importante resaltar el papel que cumple el cloruro de calcio utilizado durante el escaldado para evitar la excesiva destrucción de las paredes celulares que puede conllevar a una mayor absorción de aceite comparado con el escaldo en agua (Moyano y Pedreschi, 2006). Las enzimas pécticas afectan a las sustancias pécticas y por lo tanto a la textura de los productos; por ejemplo, la pectina metilesterasa PME es una enzima muy específica que hidroliza exclusivamente los enlaces de la cadena pectina, aumentando el número de carboxilatos R-COOH y deteriorando su dureza (Mendoza y Herrera, 2012). En este caso, tanto las enzimas como los carboxilatos disponibles deben contrarrestarse y para esto se realiza el escaldado con $\mathrm{CaCl}_{2}$. El escaldado se recomienda realizar a temperaturas mayores a $80{ }^{\circ} \mathrm{C}$ y durante un tiempo entre 2 y 7 minutos para inactivar la enzima PME y en una solución de $\mathrm{CaCl}_{2}$ para que los carboxilatos disponibles reaccionen con los iones divalentes de $\mathrm{Ca}^{+2}$ presentes en la sal utilizada y aumente el número de puentes metálicos y se refuerce la estructura de la pared celular (González-Martínez et al., 2004). Taiwo y Baik (2007) analizaron el comportamiento de las propiedades texturales luego de freír discos de batata de un centímetro de espesor a $170^{\circ} \mathrm{C}$ durante 5 minutos. Los resultados indicaron una dureza entre valores de 800 y 3000 gramos, siendo los valores del escaldado los más altos entre 2200 y 3000 gramos. Usawakesmanee et al. (2008) obtuvieron valores similares de dureza de papas apanadas con $6 \%$ de MC de $2563 \pm 144 \mathrm{~N}$ significativamente menor que las muestras sin recubrimiento de $2690 \pm 151 \mathrm{~N}$, justificado porque la muestra control había perdido mayor cantidad de humedad. 
En los valores de adhesividad no se observaron efectos significativos en ninguno de los factores ni en la interacción de estos dos factores. La adhesividad indica el área de fuerza negativa y representa la fuerza necesaria para separar el émbolo de compresión de la muestra y es también una medida de la pegajosidad de la muestra. Taiwo y Baik (2007) reportan valores de -13 a -27 g.s para muestras escaldadas y un rango total de 0 a -115 g.s para un amplio rango de pretratamientos en discos de batata. Comparados con los valores de este rango se observa que la adhesividad obtenida en este estudio es pequeña y puede considerarse muy positiva gracias a que es una característica que no es deseable en los productos fritos (Taiwo y Baik, 2007).

En los valores de cohesividad se evidencia un efecto altamente significativo de la temperatura, del recubrimiento y de la interacción de estos dos factores. La cohesividad indica la tendencia a estar más unido el producto y su menor tendencia a desintegrarse debido a una acción mecánica. Las muestras que presentaron mayor cohesividad fueron las fritas a 190 y $170{ }^{\circ} \mathrm{C}$ que las fritas a $150 \stackrel{\circ}{\mathrm{C}}$ y las muestras que se recubrieron con $\mathrm{CMC}$ frente a las que no utilizaron recubrimiento. Las muestras fritas a $150{ }^{\circ} \mathrm{C}$ presentaron la menor cohesividad y de éstas la muestra sin recubrimiento presentó el menor valor. Los tratamientos como el escaldado y el recubrimiento fortalecen la estructura del producto mediante el ligado del agua y dan cohesividad a las muestras (Taiwo y Baik, 2007). Estos resultados contrastan con el comportamiento reportado por Kassama y Ngadi (2005) ya que se espera que a altas temperaturas aumente la porosidad del producto y disminuya la cohesividad; sin embargo, estos resultados se pueden justificar debido a que cuando las muestras tienen mayor contenido de agua, en este caso las fritas a $150{ }^{\circ} \mathrm{C}$, se presenta mayor degradación hidrotérmica de la pectina de los tejidos en las paredes celulares produciendo una disminución en la estructura del parénquima de la batata (Liu y Scanlon, 2007). Taiwo y Baik (2007) reportaron valores de cohesividad entre 0.367 y 0.504 y los más altos fueron los presentados en las muestras escaldadas entre 0.49 y 0.50 .

Los factores de temperatura y de recubrimiento no mostraron un efecto significativo sobre los valores de elasticidad, gomosidad y masticabilidad. Tampoco lo hizo la interacción de los factores. Taiwo y Baik (2007) reportaron valores de elasticidad entre $48 \%$ a $63 \%$ y los altos valores se relacionan con el aumento en la porosidad del producto. También reportaron valores de masticabilidad para productos escaldados, cuyos valores entre 1300 y $1600 \mathrm{~g}$ son mayores si se comparan con el rango para otros tratamientos entre $200 \mathrm{y}$ $1600 \mathrm{~g}$. Por último, no se encontraron en la literatura valores reportados para la gomosidad de productos fritos. Se puede concluir hasta este punto que el recubrimiento aporta características deseables en los productos fritos porque los hace más sanos y podría recomendarse gracias a que no altera la dureza, la adhesividad, la elasticidad, la gomosidad y la masticabilidad; además confiere mayor cohesividad según los resultados mostrados en este estudio. Además, se ha demostrado que tiene buena barrera contra el oxígeno y brindan protección contra la oxidación lipídica e inhiben la formación de acrilamida hasta en un 30 \% (Varela y Fiszman, 2011). A mayores temperaturas de freído se acelera la formación de la costra con respecto a las temperaturas menores (Bouchon y Pyle, 2005; Alvis et al., 2008) y el rápido secado mejora las propiedades mecánicas y estructurales del producto final: rápida cocción, pardeamiento y desarrollo de la textura, el sabor y el color (Durán et al., 2007; Alvis et al., 2008). Sin embargo, en este estudio no se puede afirmar cuál de todos los tratamientos ha sido el mejor porque no se trata de una optimización en las variables de respuesta y sólo se han analizado los efectos de los factores de recubrimiento y temperatura en la textura del producto frito.

\section{CONCLUSIONES}

Las variedades tuvieron diferencias significativas en su contenido bromatológico, especialmente en el contenido de azúcares reductores, lo que permitió la selección de la batata roja para utilizarla mediante freído por inmersión. El recubrimiento y la temperatura de freído no afectaron significativamente a las variables de textura como dureza, adhesividad, elasticidad, gomosidad y masticabilidad. Pero si afectaron la cohesividad de los productos. La fuerza de corte del producto sólo se vio afectado por el uso del recubrimiento.

\section{AGRADECIMIENTOS}

Los autores agradecen el apoyo logístico y financiero prestado por la Universidad de Córdoba, a través de la oficina de Investigación y extensión.

\section{REFERENCIAS}

Agronet, [Consultado: Marzo de 2014] Disponible en http://agronet.gov.co/ (2014).

Albán, A. y L. Cadavid, Producción y usos de batata. CLAYUCA (2006). 
Alvis, A., H. Villada y D. Villada, Efecto de la temperatura y tiempo de fritura sobre las características sensoriales del ñame (Dioscorea alata). Información Tecnológica 19(5): 19-26 (2008).

An, L.V., B. Frankow-Lindberg y J.E. Lindberg, Effect of harvesting interval and defoliation on yield and chemical composition of leaves, stems and tubers of sweet potato (Ipomoea batatas L.(Lam.)) plant parts. Field Crops Research 82:49-58 (2003).

AOAC, Métodos de análisis de la asociación oficial de química analítica para determinar humedad, fibra, cenizas, grasa y proteína. Chapter 32: 1, 2, 5 y 14, Washington, U.S.A. (2003).

Arrieta, W. y R. Gutiérrez, Monografía del cultivo de la Batata (Ipomoea batatas Lam). Universidad de Córdoba. Montería. Páginas 109 (1995).

Bouchon, P. y D. Pyle, Modelling oil absorption during post-frying cooling II: Solution of the mathematical model, model testing and simulations. Food and Bioproducts Processing 83(C4): $261-272$ (2005).

Durán, M., F. Pedreschi, P. Moyano y E. Troncoso, Oil partition in pre-treated potato slices during frying and cooling. Journal of Food Engineering 81:257:265 (2007).

FAO, Producción de cultivos andinos: libros, proyectos, galería fotográfica sobre cultivos andinos. [CDROM]. También disponible en: http://www.rlc.fao.org/es/agricultura/produ/ y http://www.rlc.fao.org/es/agricultura/produ/cdrom/indice_gral.htm . (2010).

FAO, [Consultado en marzo de 2014] Disponible en http://faostat.fao.org (2014).

Farinu, A. y O. Baik, Thermal properties of sweet potato with its moisture content and temperature. International Journal of Food Properties 10: 703-719 (2007).

Gołubowska, G., Changes of polysaccharide content and texture of potato during French fries production. Food Chemistry 90: 847-851 (2005).

González-Martínez, G., L. Ahrné, V. Gekas y I. Sjöholm, Analysis of temperature distribution in potato tissue during blanching and its effect on the absolute residual pectin methylesterase activity. Journal of FoodEngineering 65: 433-441 (2004).

Hleap, J. y V. Velasco, Análisis de las propiedades de textura durante el almacenamiento de salchichas elaboradas a partir de tilapia roja (Oreochromis sp.). Facultad de Ciencias Agropecuarias 8(2): 46-56 (2010).

Iwe, M., I. Wolters, G. Gort, W. Stolp y D. Zuilichem, Behaviour of gelatinisation and viscosity in soy-sweet potato mixtures by single screw extrusion: a response surface analysis. Journal of Food Engineeting 38: 369-379 (1998).

Kassama, L. y M. Ngadi, Pore development and moisture transfer in chicken meat during deep-fat frying. Drying Technology 23: 907-923 (2005).

Kita, A., G. Lisińska y G. Gołubowska, The effects of oils and frying temperatures on the texture and fat content of potato crisps. Food Chemistry 102: 1-5 (2007).

Lisińska, G. y G. Gołubowska, Structural changes of potato tissue during French fries production. Food Chemistry 93: 681-687 (2005).

Liu, E. y M. Scanlon, Modeling the effect of blanching conditions on the texture of potato strips. Journal of Food Engineering 81: 292-297 (2007).

Mai Tran, T., X. Dong Chen y C. Southern, Reducing oil content of fried potato crisps considerably using a 'sweet' pre-treatment technique. Journal of Food Engineering 80: 719-726 (2007).

Mendoza, R. y A. Herrera, Cinética de Inactivación de la Enzima Peroxidasa, Color y Textura en Papa Criolla (Solanum tuberosum Grupo phureja) sometida a tres Condiciones de Escaldado. Información tecnológica 3 (24): $73-82$ (2012). 
Mestdagh, F., T. De Wilde, S. Fraselle, Y. Govaert, W. Ooghe, J. Degroodt, R. Verhé, C. Van Peteghem y B. De Meulenaer, Optimization of the blanching process to reduce acrylamide in fried potatoes. LWT - Food Science and Technology 41: 1648-1654 (2008).

Moyano, P. y F. Pedreschi, Kinetics of oil uptake during frying of potato slices: effect of pre-treatments. LWT 39: 285-291 (2006).

Pedreschi, F., K. Kaack, K. Granby y E. Troncoso, Acrylamide reduction under different pre-treatments in French fries. Journal of Food Engineering 79: 1287-1294 (2007).

Pletsch, A., El cultivo de la Batata. Centro Regional INTA Corrientes (2006).

Sanz, T., C. Primo-Martín y T. Van Vliet, Characterization of crispness of French fries by fracture and acoustic measurements, effect of pre-frying and final frying times. Food Research International 40:63-70 (2007).

Taiwo, K. y O. Baik, Effects of pre-treatments on the shrinkage and textural properties of fried sweet potatoes. LWT 40: 661-668 (2007).

Universidad de Córdoba, Manual de prácticas de análisis de alimentos. Serie, Guías de Laboratorios, (2010).

Tavera-Quiroz, M.J., M. Urriza, A. Pinotti y N. Bertola, Plasticized methylcellulose coating for reducing oil uptake in potato chips. Journal of the Science of Food and Agriculture, 92 (7) 1346-1353 (2012).

Usawakesmanee, W., M. Chinnan, P. Wuttijumnong, A. Jangchud y N. Raksakulthai, Effect of edible coating ingredients incorporated into predusting mix on moisture content, fat content and consumer acceptability of fried breaded product. Songklanakarin J. Sci. Technol. 30(1): 25-34 (2008).

Varela, P. y S.M. Fiszman, Hydrocolloids in fried foods. A review. Food Hydrocolloids: 1-12 (2011).

Vitrac, O., D. Dufour, G. Trystram y A. Raoult-Wack, Characterization of heat and mass transfer during deepfat frying and its effect on cassava chip quality. Journal of Food Engineering 53: 161-176 (2002).

Waramboi, J., S. Dennien, M. Gidley y P. Sopade, Characterisation of sweetpotato from Papua New Guinea and Australia: Physicochemical, pasting and gelatinisation properties. Food Chemistry 126: 1759-1770 (2011).

Zhang, L., y otros 7 autores, Application of simultaneous saccharification and fermentation (SSF) from viscosity reducing of raw sweet potato for bioethanol production at laboratory, pilot and industrial scales. Bioresource Technology 102: $4573-4579$ (2011). 